\title{
HIGH-FREQUENCY CUTOFF IN TYPE III BURSTS
}

\author{
A.A.Stanislavsky ${ }^{1,2}$, A.A.Konovalenko ${ }^{1}$, Ya.S.Volvach ${ }^{1}$, A.A.Koval ${ }^{1}$ \\ ${ }^{1}$ Institute of Radio Astronomy, Kharkiv, Ukraine, astex@ukr.net \\ ${ }^{2}$ V.N. Karazin Kharkiv National University, Kharkiv, Ukraine
}

\begin{abstract}
In this article we report about a group of solar bursts with high-frequency cutoff, observed on 19 August of 2012 near 8:23 UT, simultaneously by three different radio telescopes: the Ukrainian decameter radio telescope (8-33 MHz), the French Nançay Decametric Array (10-70 MHz) and the Italian San Vito Solar Observatory of RSTN $(25-180 \mathrm{MHz})$. Morphologically the bursts are very similar to the type III bursts. The solar activity is connected with the emergency of a new group of solar spots on the far side of the Sun with respect to observers on Earth. The solar bursts accompany many moderate flares over eastern limb. The refraction of the behind-limb radio bursts towards the Earth is favorable, if CMEs generate low-density cavities in solar corona.
\end{abstract}

Keywords: Sun: radio emission - bursts: UTR-2 radio telescope

\section{Introduction}

Sporadic radio emission of the Sun is observed in a wide range of wavelengths, from millimeters to kilometers. The radio waves emerge from different heights (layers) of the solar atmosphere: radiation of millimeter range is emitted by the lower chromosphere, $\mathrm{cm}$-waves are produced by the middle and upper chromosphere, decimeters originate from the transition region, meters come from the lower corona, and the radio emission in the decameter range is generated by the upper corona and so on (Zheleznyakov, 1970). An important component of solar activity is various types of solar bursts. Solar bursts can serve as a probing signal to study properties of solar corona plasma. Multi-frequency observations of solar radio emission allow ones to determine the electron density, temperature and magnetic field strength in the solar corona at different heights.

In the decameter range the solar activity are observed in the type III bursts associated with IIIb bursts, $\mathrm{U}$ and J bursts, the type II and IV bursts, striae, drifting pairs and S-bursts as well as other events (Krüger, 1979). They occur with varying frequency of occurrence. The main parameters characterizing the bursts are the frequency drift rate, instantaneous bandwidth, duration, flux, polarization, etc. All these parameters indicate the type of solar bursts. The type III bursts is the most numerous collection of solar bursts recorded in radio observations. They occur on the whole Sun. However, the most of observations are performed by ground-based instruments, and a significant part of solar radio events is unavailable for the radio astronomy tools. This situation is improved by space mis- sions such as WIND and STEREO, but they are not as sensitive as the newest radio telescopes LOFAR, UTR-2, GURT and others. Nevertheless, some solar bursts from the far side of the Sun can arrive at ground-based telescopes due to specific conditions in solar corona. Such events are related to solar bursts with high-frequency cutoff. Recall, the low-frequency cutoff (radio emission below $10 \mathrm{MHz}$ cannot reach to ground-based instruments) in cosmic radio emission appears due to the ionosphere. As for the high-frequency cut-off, its cause is totally different. This effect is explained by the burst's source position in solar corona with respect to the observer. Really, if the source is moved radially from behind the solar limb, it may be occulted by solar corona. Therefore, the most high-frequency part of the solar burst spectrum is reflected away from the direction of the Earth. The propagation of radio emission of behind-limb solar bursts in the solar corona imposes a number of features on the characteristics of these bursts for terrestrial observers.

In this work we present preliminary results related to the observations of solar bursts with high-frequency cutoff (Fig.1), demonstrating clearly the properties typical for the type III ones. The identification is useful for understanding their nature and frequency-time evolution.

\section{Instruments and observations}

On 19 August of 2012 starting with 8:23 UT the Ukrainian decameter radio telescope UTR-2 (8-33 MHz) has registered about a dozen of solar bursts following each other. Their main peculiarity was high-frequency cutoff near to the upper boundary frequency of observations for UTR-2. Note that we used only four sections of the northsouth antenna array of this radio telescope. The total effective area of such an antenna part is $50000 \mathrm{~m}^{2}$, and the beam pattern size is $1^{\circ} \times 15^{\circ}$ at $25 \mathrm{MHz}$. The solar radio data were recorded by the digital DSP spectrometer operating in the frequency range of 9-33 $\mathrm{MHz}$ with the time resolution of $100 \mathrm{~ms}$ and frequency resolution of $4 \mathrm{kHz}$. Some of these solar bursts had their cutoff frequency above frequency capabilities of UTR-2. To get a full view of all the events, we have studied the observations of two different radio telescopes at the same time: the French Nançay Decametric Array $(10-70 \mathrm{MHz})$ and the Italian San Vito Solar Observatory of RSTN (25-180 MHz). Both the instruments also show the registration the given bursts. This allowed us to find the frequency of cutoff of each bursts in the collection. They were different from burst to burst and lie in the range of 30-55 MHz. The simultaneous 
observations in different parts of the world indicate that the given bursts and their characteristic features have solar origin rather than either ionospheric or instrumental links.

The frequency drift rate of the solar bursts was negative, 1.1-3.3 MHz/s that corresponds to the characteristic values as applied to the type III bursts in decameter wavelengths. The same is related to their duration. The flux of the set does not exceed 1000 s.f.u. ( 1 s.f.u. $=10^{-22} \mathrm{~W} \mathrm{~m}^{-2} \mathrm{~Hz}^{-1}$ ) that points to the moderate character of bursts in intensity.

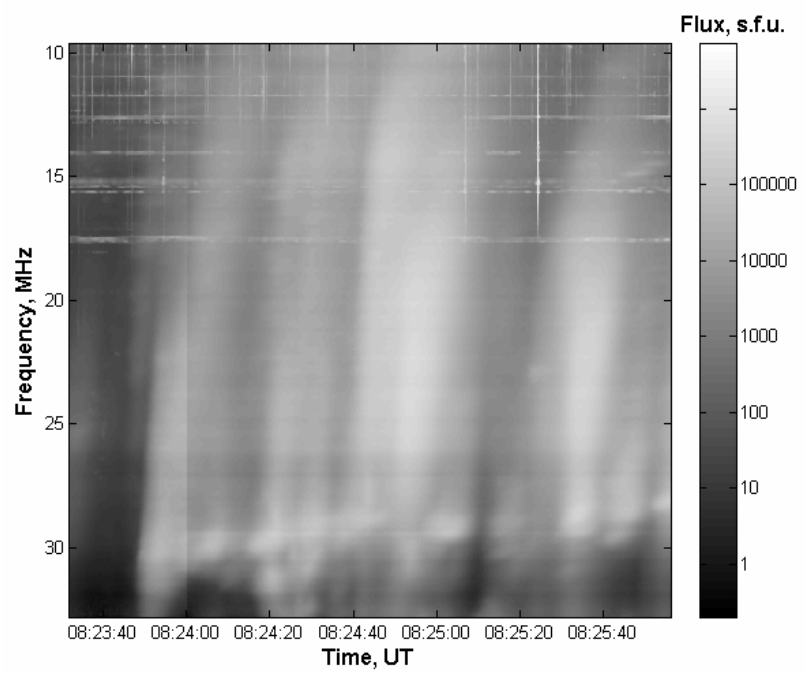

Figure 1: Dynamical spectrum of solar radio bursts with high-frequency cutoff, observed on 19 Augusts of 2012 after 8:23 UT by the telescopes UTR-2. Horizontal bright lines are disturbances due to broadcasting radio stations. Vertical lines in the range about $8-18 \mathrm{MHz}$ indicate terrestrial lightning discharges.

In this connection it should be mentioned that recently a solar burst with high-frequency cutoff in the decameter range of wavelengths has been described by Brazhenko et al. (2012). This was a single radio event on 3 June of 2011. It had the cutoff at $\sim 27.5 \mathrm{MHz}$, the half-power duration $17-22 \mathrm{~s}$, the frequency drift rate $100-500 \mathrm{kHz} / \mathrm{s}$. Moreover, the dynamical spectrum of this event demonstrates an unusual fine structure with filaments called "caterpillar". The unique properties of this burst make it difficult to classify, according to the conventional scheme of types of solar bursts. Therefore, the further patrol of new events with high-frequency cutoff is very important for a successful interpretation of the phenomena.

\section{Interpretation}

As it has been shown by Melnik et al. (2012), one of plausible interpretations, explaining features of the unusual burst on 3 June of 2011 , is that the origin of the event came from behind the solar limb, and the cutoff of its radio emission appeared because the high-frequency part of the burst's spectrum does not pass towards the Earth. This approach also can be applied for the group of solar bursts observed on 19 August of 2012. Really, the solar activity was connected with a new group of solar spots (AR 11548, about N19E87) born 17 August 2012 on the far side of the Sun for terrestrial observers. This is supported by the STEREO observations (see http://stereossc.nascom.nasa.gov/data.shtml). However, the plasma dispersion of solar corona influences on the direction of radio wave propagation that can lead to the reflection of radio rays away from the Earth rather than their refraction towards the Earth (see, for example, Thejappa and MacDowall, 2010). Consequently, the purely spherical model of solar corona density is not favorable for behind-limb solar bursts to detect them by ground-based tools. The situation may be rescued by coronal mass ejections (CMEs) associated with the post-limb flare activity. In particular, the CMEs generate lower-density cavities in solar corona (Gibson S.E. et al., 2010) through which the radio emission of behind-limb bursts can fall to Earth. This problem will be considered in more detail elsewhere.

\section{Conclusions and future work}

In this work we have shown that the solar bursts with high-frequency cutoff can be identified as usual type III bursts. Because of various effects of radio wave propagation in solar corona (generally speaking, the latter is an inhomogeneous medium), being originally the type III bursts, the behind-limb bursts are distorted. Maybe that is why the bursts on 3 June of 2011 looks like "caterpillar". Anyway, the analysis of ray tracing in inhomogeneous solar corona for the solar bursts with cutoff requires a separate examination. Additionally, notice that it would be useful to check the functional evolution of frequency drift rates and durations in frequency for the events on $19 \mathrm{Au}-$ gusts of 2012 considered in this paper. According to Alvarez and Haddock (1973), as well as Elgarøy and Lyngstad (1972), there are characteristic dependences of the frequency drift rate and the duration in frequency, supported by ordinary type III bursts. It would be useful to check them as applied to the bursts of this paper in future.

Acknowledgements. This research was supported particularly by Research Grant "Synchronized simultaneous study of radio emission of solar system objects by lowfrequency ground- and space-based astronomy" from National Academy of Sciences of Ukraine. We want to thank the STEREO and SOHO teams for developing and operating the instruments as well as for their open data policy.

\section{References}

Alvarez H., Haddock F.T.: 1973, Sol. Phys., 29, 197.

Brazhenko A.I. et al.: 2012, RPRA, 3, 279.

Elgarøy Ø., Lyngstad E.: 1972, $A \& A, \mathbf{1 6}, 1$.

Gibson S.E. et al.: 2010, ApJ, 724, 1133.

Krüger A.: 1979, Introduction to Solar Radio Astronomy and Radio Physics, Springer, Berlin.

Melnik V.N. et al.: 2012, RPRA, 17, 199 (in Russian).

Thejappa G., MacDowall R.J.: 2010, ApJ, 720, 1395.

Zheleznyakov V.V.: 1970, Radio Emission of the Sun and Planets, Pergamon Press, Oxford. 